Schmerz 2021 · 35:379-381

https://doi.org/10.1007/s00482-021-00604-y

Angenommen: 19. Oktober 2021

(c) Der/die Autor(en) 2021

\title{
Schmerzgesellschaft koordiniert inzwischen drei Innovationsfondsprojekte
}

Winfried Meißner

Sektion Schmerzmedizin, Klinik für Anästhesiologie und Intensivmedizin, Abt. für Palliativmedizin, Klinik für Innere Medizin II, Universitätsklinikum Jena, Jena, Deutschland

Das Einwerben von Drittmittelprojekten gehört zum täglichen Alltag von Wissenschaftlern/-innen. Dabei gilt: Je unabhängiger der Auswahlprozess des Drittmittelgebers, je größer die Konkurrenz und je größer der Projektumfang, desto schwieriger ist es, einen erfolgreichen Antrag auch „durchzubekommen". Eine erfolgreiche Einwerbung eines kompetitiven und multizentrischen BMBF-, DFG- oder EUProjekts als Hauptantragsteller/-in ist ein Meilenstein und bisweilen Höhepunkt in jeder wissenschaftlichen Karriere. Und gerade bei großen Verbundprojekten zählt nicht nur die wissenschaftliche Exzellenz: Auch das Projektmanagement ist ein hoch komplexer und anspruchsvoller Prozess, angefangen vom Planen und Erstellen des Antrags, der Vertrags-, Finanz- und Personalplanung, Controlling, Reporting, Auditing, der Öffentlichkeits- und Disseminationsarbeit bis zum Erstellen der Abschlussberichte. Daher ist es nicht verwunderlich, dass solche Projekte federführend meist aus Hochschulen bzw. akademischen Einrichtungen mit entsprechender organisatorischer Infrastruktur stammen.

Umso erfreulicher ist es, dass die Deutsche Schmerzgesellschaft e. V. bereits das dritte Innovationsfondsprojekt als Hauptantragstellerin erfolgreich eingeworben und dabei das Projektmanagement übernommen hat: Nach PAIN2020 und POETPAIN nun auch PAIN 2.0 (https://www. schmerzgesellschaft.de/wissenschaft). Der Innovationsfonds basiert auf dem 2015 verabschiedeten Versorgungsstärkungsgesetz. Gesundheitspolitisches Ziel war und ist die Stärkung sektorenübergreifender Versorgungsformen und Versorgungsforschung und die Bereitstellung eines entsprechenden Innovationsbud- gets auch aus Mitteln der Gesetzlichen Krankenversicherung (GKV). Träger ist der Gemeinsame Bundesausschuss (G-BA), die Projektabwicklung erfolgt, wie bei vielen anderen Projekten auch, durch das Deutsche Zentrum für Luft- und Raumfahrt (DLR). Die Anträge wurden unabhängig begutachtet und die Förderquoten (2019/2020 zwischen $18 \%$ und $38 \%$ ) zeigen, wie kompetitiv der Auswahlprozess ist.

Traditionell betrachten Fachgesellschaften das Einwerben von Drittmittelprojekten nicht als ihre Hauptaufgabe. Die Deutsche Schmerzgesellschaft sah jedoch im Innovationsfonds eine große Chance, relevante schmerzmedizinische Versorgungsthemen zu adressieren, die nicht im Fokus herkömmlicher Förderinstrumente stehen. In PAIN2020 und PAIN 2.0 wird die Bedeutung eines interdisziplinären Assessments und einer ambulanten interdisziplinär-multimodalen Schmerztherapie bei Patienten mit länger anhaltenden Schmerzen evaluiert. Dabei soll untersucht werden, ob durch derartige relativ frühzeitige Interventionen funktionale Versorgungswege eingeschlagen und eine Chronifizierung verhindert werden kann. Auch das Projekt POET-PAIN untersucht den Übergang von akuten zu chronischen Schmerzen, jedoch im perioperativen Bereich: Patienten mit Risikooperationen sollen von einem interdisziplinären Team perioperativ gescreent und begleitet werden, Ziel ist auch hier eine Reduktion der chronischen Schmerzen, die nach einer Operation entstehen können.

Solche versorgungswissenschaftliche prospektive Fragestellungen standen bisher weder im Mittelpunkt traditioneller 
Förderinstrumente noch universitärer Forschergruppen. Daher ist es zu begrüßen, dass in der Deutschen Schmerzgesellschaft e. V. in relativ kurzer Zeit ein hoch professionelles Team aufgebaut wurde, das nicht nur die Antragserstellung und die Projektabwicklung betreut, sondern auch in der gesundheitspolitischen Szene gut vernetzt ist. Dieses Team ist vergleichbar mit einem vollwertigen Wissenschaftsmanagement-Office und stellt eine wertvolle Ressource für zukünftige Anträge als auch für viele andere Aktivitäten der Gesellschaft dar.

Warum beantragen Wissenschaftlerinnen und Wissenschaftler ihr Projekt über eine Fachgesellschaft? Auch in vielen Universitäten ist es für Forscher und Forscherinnen nicht ganz einfach, Zugang zu den unterstützenden Ressourcen für große Projektanträge und deren Abwicklung zu erhalten - sofern diese überhaupt vorhanden sind. Nicht selten läuft das Antragsgeschäft über die Lehrstuhlinhaber/-innen oder etablierte Forschungsgruppen, und gerade Nachwuchsforscherinnen und -forscher haben gewisse Barrieren zu überwinden. Auch sehr starke Forschungsschwerpunkte können dazu führen, dass "freie“ Themen in einer Universität weniger gefördert werden. Ein weiterer Vorteil kann das weitverzweigte, themenaffine und landesweite Netzwerk der Mitglieder einer Fachgesellschaft sein, das das Finden von Kooperationspartnern erleichtert.

Aber auch für unsere Gesellschaft stellen sich einige wichtige Fragen: Wer bekommt den Zugang zu den wissenschaftsfördernden Ressourcen der Fachgesellschaft? Welche Anträge sollen priorisiert werden? Wie wollen wir mit konkurrierenden Ideen umgehen? Wofür wird der nicht unbeträchtliche Overhead eingesetzt, und wer entscheidet darüber? Und kann es eine Gefahr für die forschungspolitische "Neutralität" einer Fachgesellschaft darstellen, wenn sie selbst eine Konkurrentin an den Drittmittelfuttertrögen wird oder ihre Anträge bestimmte Forschungsspektren nicht abdecken? Das Präsidium unserer Gesellschaft wird sich diesen Fragen stellen, und alle Mitglieder sind herzlich eingeladen, sich an diesem Diskurs zu beteiligen.

Ungelöst ist allerdings bisher die Frage, wie die "Lorbeeren“ in Form von leis- tungsorientierten Mitteln (LOM) an der Herkunftsinstitution der hauptverantwortlichen Wissenschaftler gewertet werden, wenn als Projektkoordinator nicht ihr Name und ihre Institution, sondern eine Fachgesellschaft und deren Präsidentin und Präsident stehen. Und auch für diese stellt es eine große Verantwortung dar, das beantragte Projekt zu begleiten und - zumindest partiell - mit zu verantworten.

\section{》) Drittmittelprojekteinwerbung durch wissenschaftliche Fachgesellschaften: bisher in Deutschland selten}

Drittmittelprojekteinwerbung primär durch wissenschaftliche Fachgesellschaften: Ein bisher in Deutschland eher seltenes Phänomen, das jedoch sowohl für Wissenschaftler als auch für die Gesellschaften neuartige Chancen bietet. Dabei sollten die Besonderheiten dieses Ansatzes gezielt genutzt werden. Aus meiner Sicht besteht eine solche Chance auch darin, diese Ressourcen für Arbeitsgruppen ohne guten Zugang zu Projektmanagementressourcen, insbesondere auch für unsere Kolleginnen und Kollegen in den Praxen, Ambulanzen und nichtuniversitären Kliniken zu öffnen, die täglich mit den drängenden klinischen Fragen konfrontiert werden. Aus ihren Kreisen kommen immer wieder klinisch hoch relevante Forschungsideen, die bisher ohne realistische Chance auf Realisierung waren.

Last but not least: Ohne die wissenschaftlichen Köpfe hinter den Anträgen - zu nennen wären hier u.a. Ulrike Kaiser, Frank Petze, Hans-Raimund Casser, Bernd Nagel, Esther Pogatzki-Zahn, Thomas Kohlmann, Nadja Nestler, Irmela Gnass, Axel Schäfer und viele andere mehr -, das Commitment der BARMER (Ursula Marschall) und das Forschungs-Office der Geschäftsstelle der Deutschen Schmerzgesellschaft e. V. - Thomas Isenberg, Carolin Martin, Katharina Augustin, Gabi Lindena, Anja Waidner, André Möller, Kevin Schulz, Christina Grams, Beate Schlag - hätte dieses Editorial nicht erscheinen können: Ganz herzlichen Dank!

Winfried Meißner
Korrespondenzadresse

Apl. Prof. Dr. Winfried Meißner

Sektion Schmerzmedizin, Klinik für

Anästhesiologie und Intensivmedizin, Abt. für

Palliativmedizin, Klinik für Innere Medizin II,

Universitätsklinikum Jena

Am Klinikum 1, 07747 Jena, Deutschland

Winfried.Meissner@med.uni-jena.de

Interessenkonflikt. W.Meißner:Vortragstätigkeiten/ Beratung: Mundipharma, Grünenthal, Bionorica, Kyowa, TAD, Tilray, Spectrum Therapeutics, Septodont, Northern Swan. Aktive Tätigkeiten in Arbeitskreisen und Kommissionen: Deutsche Schmerzgesellschaft, IASP. Institutionelle Forschungsförderung: EU, DFG, BMBF, EFIC, Pfizer, Mundipharma, Grünenthal. 
Hier steht eine Anzeige.

黑 Springer 